Case Report

\title{
Right Heart Failure in Young People in Emerging Countries Is Most Commonly Secondary to Tuberculous Constrictive Pericarditis
}

\author{
Charbel Naim $^{1}$, Simon Bejjani ${ }^{2}$, Omar Boustros $^{2}$, Moussa Abi Ghanem², Nader Wansa ${ }^{1}$, \\ Pierrette Habib $^{1}$, Nadine Kawkabani ${ }^{3}$, , Roula Darwish ${ }^{3}$, Paul Charbel ${ }^{1}$, Bassam Abou Khalil ${ }^{2}$ \\ ${ }^{1}$ Department of Cardiology, Saint George Hospital- University Medical Center, University of Balamand, Beirut, Lebanon \\ ${ }^{2}$ Department of Cardiothoracic Surgery, Saint George Hospital- University Medical Center, University of Balamand, Beirut, Lebanon \\ ${ }^{3}$ Department of Cardiac Anesthesia, Saint George Hospital- University Medical Center, University of Balamand, Beirut, Lebanon
}

Email address:

nadine_kaoukabani@yahoo.com (N. Kawkabani)

${ }^{*}$ Corresponding author

To cite this article:

Charbel Naim, Simon Bejjani, Omar Boustros, Moussa Abi Ghanem, Nader Wansa, Pierrette Habib, Nadine Kawkabani, Roula Darwish, Paul Charbel, Bassam Abou Khalil. Right Heart Failure in Young People in Emerging Countries Is Most Commonly Secondary to Tuberculous Constrictive Pericarditis. International Journal of Cardiovascular and Thoracic Surgery. Vol. 5, No. 1, 2019, pp. 6-11.

doi: $10.11648 /$ j.ijcts.20190501.12

Received: December 9, 2018; Accepted: December 25, 2018; Published: April 10, 2019

\begin{abstract}
Constrictive pericarditis is a rare disease with a challenging diagnosis and treatment. Tuberculous pericarditis accounts to $1-2 \%$ of the cases in the United States, but is the most common cause in other countries. We report a 17-year-old male presenting with right heart failure. His initial work up that included an Electrocardiogram, chest roentgenogram and echocardiogram suspected constrictive pericarditis. Right heart catheterization and cardiac MRI confirmed the diagnosis. Total Pericardiectomy was performed. Tissue PCR showed tuberculous pericarditis and the patient was treated with anti-tuberculous drugs for 9 months. Tuberculosis is a common cause of constrictive pericarditis especially in developing countries, whereas in developed countries most cases are idiopathic. To note that the diagnosis of tuberculous pericarditis can be difficult especially in negative PPD patients However It remains a common cause of constrictive pericarditis in some parts of the world where a high index of suspicion is requested. The treatment is curative and thus an aggressive approach in diagnosis and treatment is indicated.
\end{abstract}

Keywords: Tuberculous Constrictive Pericarditis, Right Heart Failure, Emerging Countries

\section{Introduction}

Constrictive Pericarditis is a relatively uncommon entity in contemporary practice. It is caused by fibrosis and calcification of the pericardium, leading to inhibition of diastolic filling of the heart. Clinical signs and symptoms are often vague. The diagnosis is challenging because it is necessary to differentiate this pathology from restrictive cardiomyopathy and other causes of right sided heart failure, such as pulmonary embolism, pulmonary hypertension, right ventricular infarction, mitral stenosis, and left ventricular systolic dysfunction. Accurate diagnosis is crucial because surgical intervention can provide complete relief of symptoms.
[1] The majority of cases in the United States are of unknown etiology, but around $10 \%$ are viral, radiation induced, rhumatic, metabolic or tuberculous in origin. However in developing countries, tuberculosis is by far the most common cause of pericardial constriction.

\section{Case Report}

We report a 17-year-old male patient who was born and raised in Baghdad, Iraq, referred to us for intermittent jaundice and possible constrictive pericarditis. The patient reported bouts of painless episodic icteric sclera without fever of one year duration. He had good exercise tolerance without chest 
symptoms and no increase in abdominal girth or lower limb edema. His mother reported a history of pneumonia at the age of 12 days for which he was hospitalized. He has no other family history of cardiac illnesses or sudden cardiac death.

On Physical exam He was afebrile with a heart rate of 80 bpm, and a blood pressure of 140/90 $\mathrm{mmHg}$. His jugular veins were dilated, but otherwise his examination was unremarkable.

His laboratory evaluation was significant for a total bilirubin of $2.0 \mathrm{mg} / \mathrm{dl}$, direct bilirubin $0.67 \mathrm{mg} / \mathrm{dl}$, alanine aminotranseferase (ALT) $21 \mathrm{U} / \mathrm{L}$, gamma glutamyl transferase (GGT) $41 \mathrm{U} / \mathrm{L}$, alkaline phosphatase (ALP) $72 \mathrm{U} / \mathrm{L}$, albumin
$4.7 \mathrm{~g} / \mathrm{dl}$, Fe $65 \mu \mathrm{g} / \mathrm{dl}$ and a normal TSH. His PPD test was negative.

His electrocardiogram showed sinus rhythm with normal intervals and diffuse non-specific repolarization abnormalities.

A Chest Roentgenogram identified a normal lung parenchyma and calcifications surrounding the heart.

An echocardiogram was done next and showed a normal left ventricular systolic function, mild mitral regurgitation, mildly dilated left atrium, pericardial thickening and findings that are compatible with some constrictive pathology." Figure $1-2-3 "$.

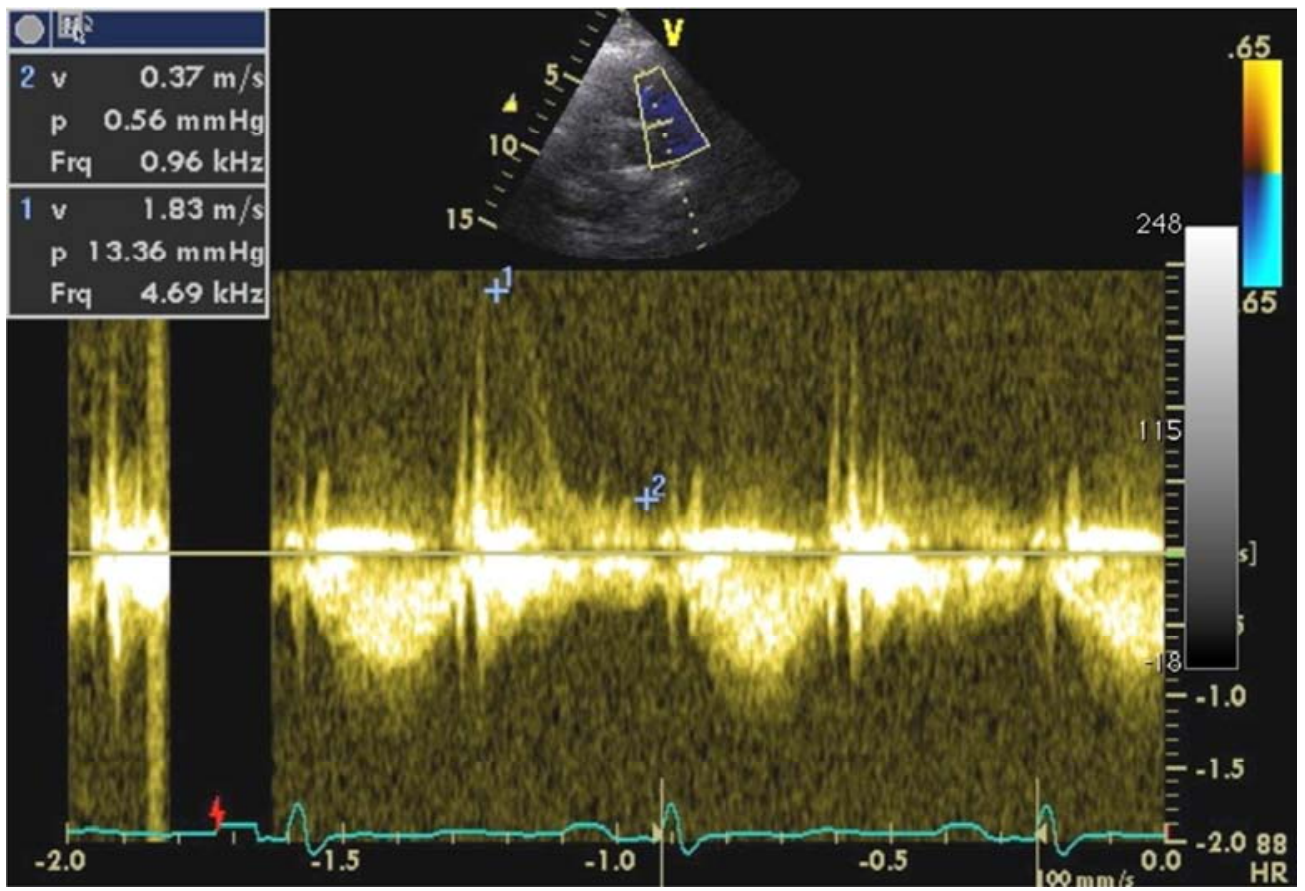

Figure 1. Pulmonary regurgitant flow shows evidence of a dip in the RV-PA diastolic pressure gradient.

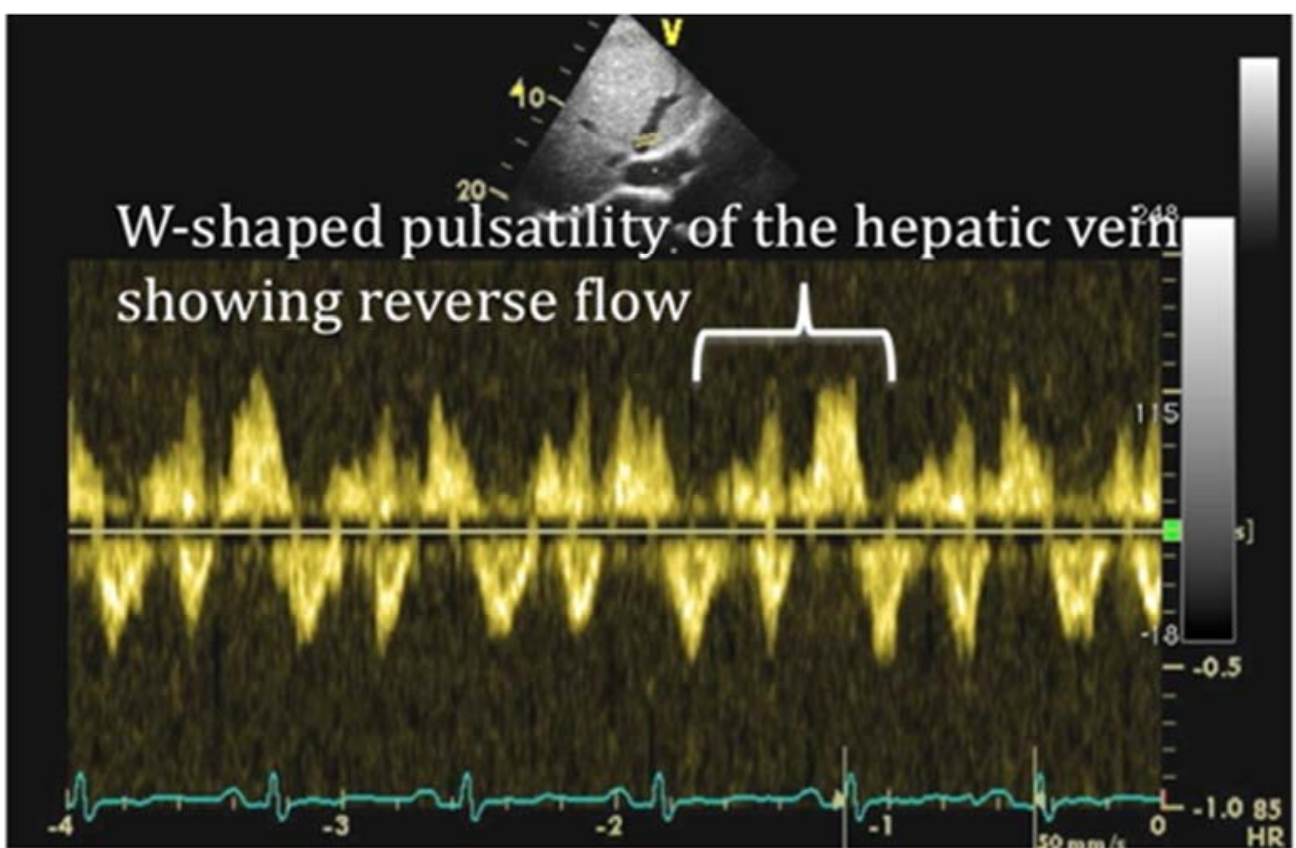

Figure 2. W Shaped pulsatility of the hepatic vein showing reverse flow. 


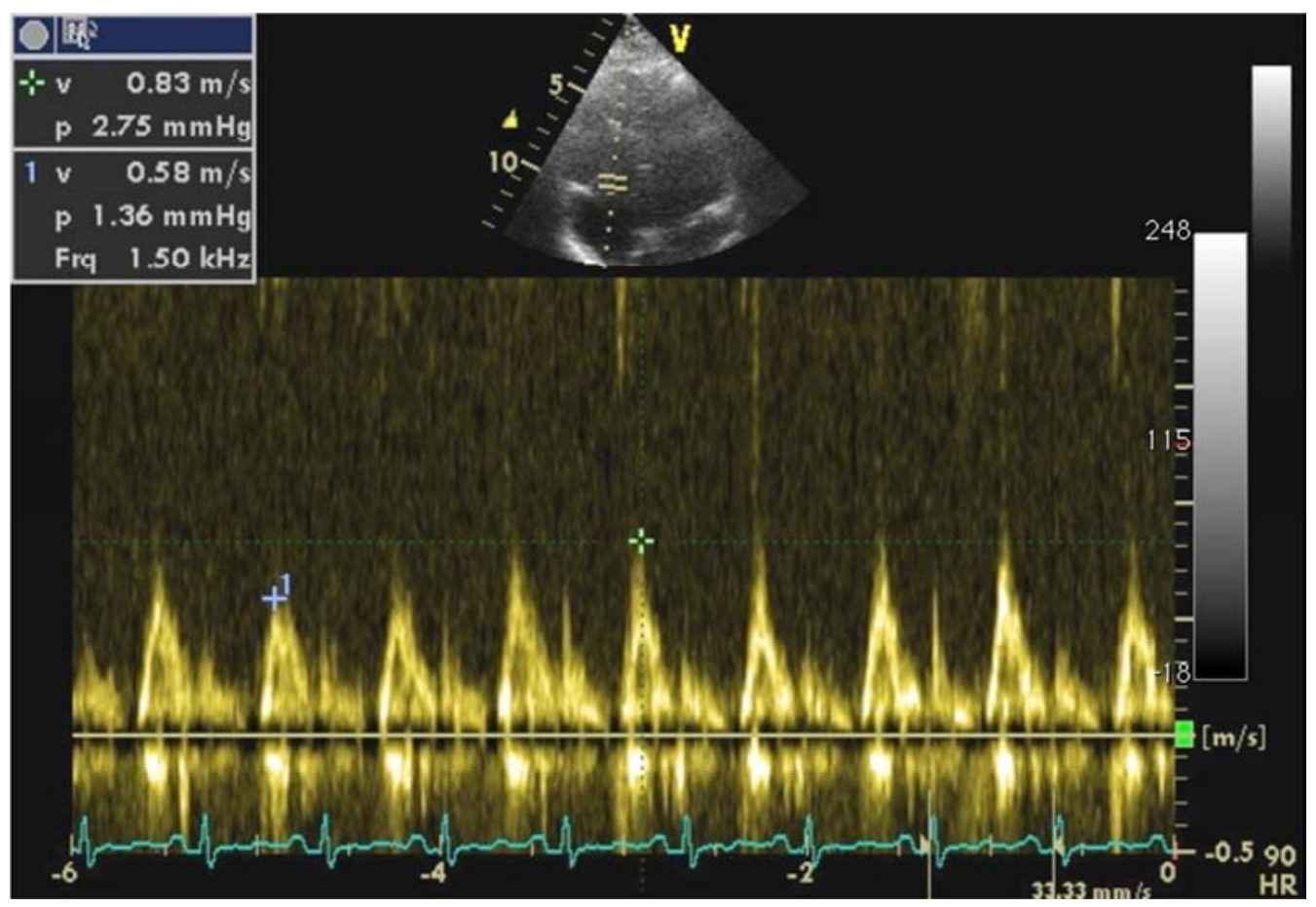

Figure 3. Respiratory variations of the tricuspid forward flow.

An abdominal ultrasound showed signs of hepatic congestion.

A right heart catheterization was performed that revealed the following:

-Right atrial waveforms with prominent $\mathrm{x}$ and $\mathrm{y}$ descents

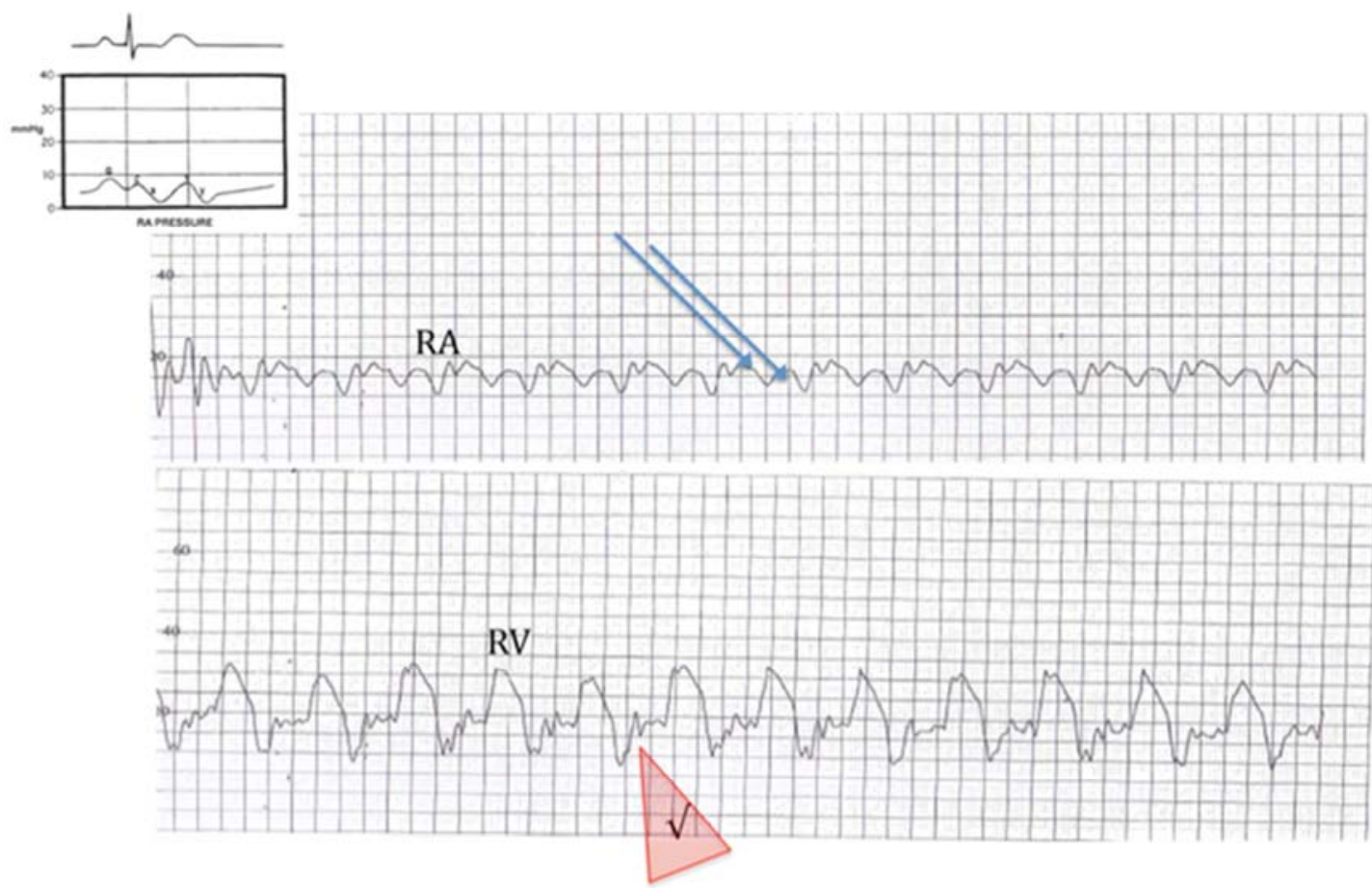

Figure 4. Square root sign.
Finally a cardiac MRI showed a thickened pericardium and a rigid aspect which was more pronounced on the free border of the right ventricle and base of the LV. Pericardial calcifications were noted with absence of pericardial effusion. The inferior vena cava was markedly dilated, with a large and
-Equilibration of the PA and RV and RA diastolic pressures -Dip and Plateau configuration of the right ventricular pressure waveform or what is known as the "square root sign" "figure 4". ested liver. On moving frames, abnormal diastolic motion of the interventricular septum with septal bounce or shutter was appreciated as well as a normal left ventricular ejection fraction."Figure 5". 


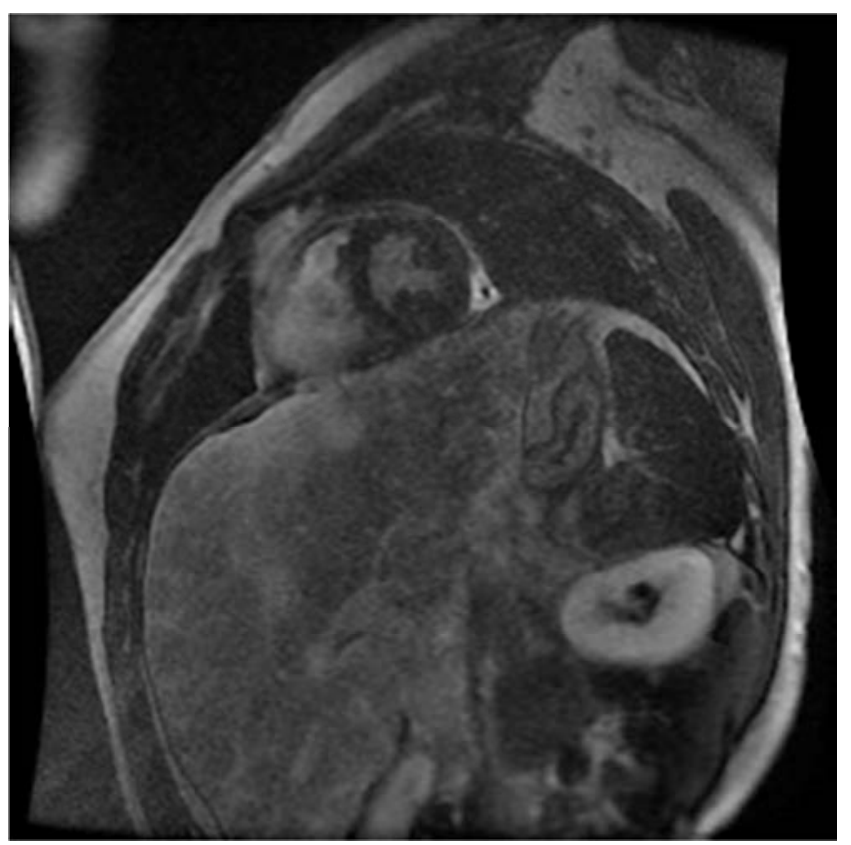

Figure 5. Cardiac MRI that shows a thickened pericardium and pericardial calcifications.

All the above confirmed a diagnosis of constrictive Pericarditis. Initially he was started on diuretics, and an ACEI. A total pericardiectomy was then performed using a median sternotomy approach. The left ventricle was decorticated first and then the right side in the usual fashion."figure6-7".

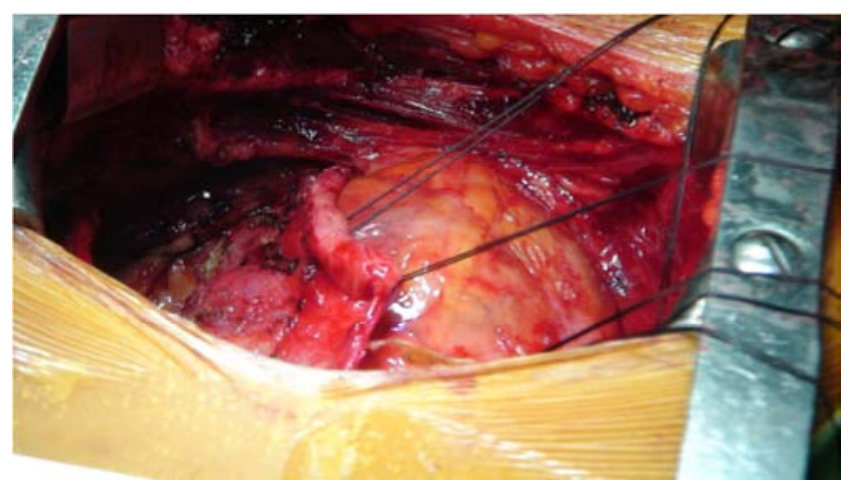

Figure 6. Stay sutures retracting the thickened pericardium.

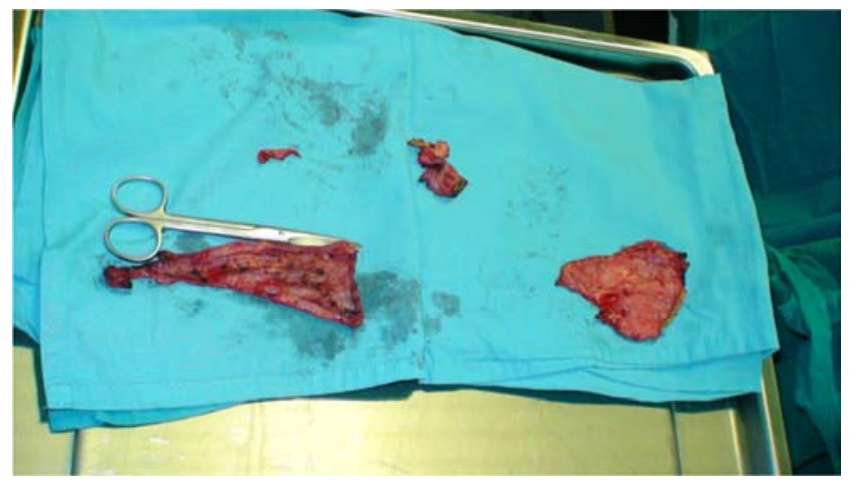

Figure 7. Total pericardiectomy showing a thick pericardium.

Post operatively, the wedge pressure and the right heart waveforms normalized. The pathologic diagnosis was fibrotic pericardial tissue. The tissue PCR was positive for Mycobacterium tuberculosis. The patient did well and was discharged home in 4 days.

The patient received 9 months of rifampin, isoniazid, ethambutol and pyrazinamide. On his one year follow-up the patient was leading a normal and healthy life .

\section{Discussion}

This is the classic form of pericardial constriction due to calcific tuberculous pericarditis an entity that has become infrequent. Tuberculosis was once a common cause of constrictive pericaditis, whereas nowadays most cases are idiopathic [2]. No single approach should be used to diagnose all cases of constrictive pericarditis. There are at present no unified recommendations for the diagnosis and management of tuberculous pericarditis [3]. The diagnostic clue is to have a high index of suspicion in a patient with right sided heart failure symptoms and signs that are disproportionate to pulmonary or left heart disease . Echocardiography may detect areas of increased echogenicity. Pericardial thickening may be asymetrical:a thorough evaluation should cover all the available windows. The classical $\mathrm{M}$ mode findings are: The multiple dense parallel lines posterior to the LV endocardium, The interventricular septal bounce :A brief posterior motion of the interventricular septum in early diastole followed by paradoxical and then a normal motion, abnormal motion of the posterior wall with an early diastolic recoil and an interventricular septum shift with inspiration which is a manifestation of both the dissociation of intrathoracic and intracardiac pressures and the interventricular dependence. On the other hand the following doppler signs are very crucial:

1-On the mitral side: A restrictive filling pattern $\mathrm{E}>\mathrm{A}$, short deceleration time, tissue Doppler analysis with elevated E' on the lateral aspect and the presence of annulus reversus(E'septal>. E'lateral).

2-On the tricuspid side a restrictive filling pattern with an increased inspiratory variation of velocities

3(-Dip) plateau of the pulmonary regurgitant jet.

4-Hepatic vein flow :Early expiratory reverse flow with a prominent atrial reversal

5 -The inferior vena cava is usually dilated .

So a mildly dilated RV, dilatation of the inferior vena cava with absence of respiratory variations in addition to the septal motion, (the deep) plateau of the pulmonary regurgitant jet and expiratory hepatic reverse flow are all in favor of constrictive pericarditis [4].

Right heart catheterization shows deep rapid Y descent corresponding clinically to friedrich sign which is a diastolic collapse of the cervical veins due to adhesion of the pericardium. Initial dip reflecting iso-volumetric relaxation and the plateau the cessation of flow after early brisk diastolic filling. Dip-and-plateau configuration of the ventricular waveforms, may be absent if the heart rate is rapid or in cases of hypovolemia where infusion of normal saline will reveal the square root sign [5].

In our case, most of these features were reported confirming 
the diagnosis of constrictive pericarditis.

On the other hand, There are several forms of constrictive pericarditis which impose different types of treatment. A cardiac MRI is indicated to ascertain the diagnosis and delineate the anatomy It is a sensitive method of measurement of pericardial thickening. In the present case, a MRI confirmed the diagnosis of constrictive pericarditis and showed a thickened pericardium with a rigid aspect especially on the free border of the right ventricle and the base of the left ventricle. These findings helped in achieving acomplete surgical procedure with an excellent outcome.
Tuberculous pericarditis affects $1-2 \%$ of patients with Tb by direct extension from mediastinal lymph nodes and occasionally by hematogenous spread. It nearly always occurs in association with another focus of $\mathrm{Tb}$, but occasionally the other focus is silent. The incidence has been decreasing, but it is still prevalent in third world countries. In fact a study done by Choundhurg et al reported395 cases of constrictive pericarditis. $88,9 \%$ of the cases were caused by tuberculosis. A higher incidence of tuberculous constrictive pericarditis was reported in a study done by Cinar et al in turkey [6] "Table 1".

Table 1. Pericardiectomy for constrictive pericarditis in the developing world: aetiology, mortality and predictors of mortality.

\begin{tabular}{|c|c|c|c|c|}
\hline Author, period & Region & $\begin{array}{l}\text { Number of } \\
\text { cases }\end{array}$ & Aetiology & $\begin{array}{l}\text { Peri-operative } \\
\text { mortality (\%) }\end{array}$ \\
\hline Bashi, 1954-85 & India & 118 & TB $(61 \%)$ & 16 \\
\hline Cinar, 1990-05 & Turkey & 70 & TB $(100 \%)$ & 8.6 \\
\hline Lin, 2005-10 & China & 51 & $\begin{array}{l}\text { TB }(65 \%) \text {, idiopathic }(25 \%) \text {, prior cardiac surgery }(6 \%) \text {, CTD }(2 \%) \text {, } \\
\text { post-trauma }(2 \%)\end{array}$ & 3.9 \\
\hline Arsan, 1983-93 & Turkey & 105 & TB $(38 \%)$, malignancy $(15 \%)$, uraemia $(11.4 \%)$, CTD $(10.5 \%)$ & 10.5 \\
\hline Chowdhury, 1984-2004 & India & 395 & TB $(88.9 \%)$, purulent $(6.3 \%)$, idiopathic $(3.7 \%)$, malignancy $(1 \%)$ & 7.6 \\
\hline
\end{tabular}

Table 1. Continued.

\begin{tabular}{|c|c|c|c|}
\hline Author, period & Late mortality (\%) & Predictors of peri-operative mortality & Predictors of late mortality \\
\hline Bashi, 1954-85 & \multirow{2}{*}{$\begin{array}{l}\overline{10} .1 ; 5 \mathrm{yr} \\
17.6 ; 10 \mathrm{yr}\end{array}$} & Pre-operative NYHA III and IV & - \\
\hline Cinar, 1990-05 & & Ascites, duration of symptoms & Ascites \\
\hline Lin, 2005-10 & $6.3 ; 1 \mathrm{yr}$ & - & $\begin{array}{l}\text { High ESR, elevated creatinine, } \\
\text { LCOS, pleural effusion }\end{array}$ \\
\hline Arsan, 1983-93 & & & - \\
\hline Chowdhury, 1984-2004 & & $\begin{array}{l}\text { *High RAP, hyperbilirubinaemia, AF, renal dysfunction, pericardial } \\
\text { calcification, thoracotomy approach, partial pericardiectomy }\end{array}$ & \\
\hline Mutyaba, 1990-2014 & & NYHA IV symptoms, hyponatraemia & \\
\hline
\end{tabular}

The disease process starts with a serosanguineous effusion, then an effusive constrictive phase that finally leads to constriction. The diagnosis is usually made by tissue PCR, or smear/culture of the pericardial fluid. Usually the patients are PPD positive, but they do not have to be especially if they are anergic.

The management of tuberculous pericarditis consists of the administration of the triple-drug antituberculous therapy for a minimum of 9 months. 5 This is the case because the mortality rate is $50 \%$ in 2 years and $80 \%$ in 5 years if untreated. In case of treatment failure or recurrence, 3 months of corticosteroid therapy may be a useful adjunct. In fact many studies have showed that steroids still have a beneficial role in the management of tuberculous constrictive pericarditis in HIV negative patients it may prevent the progression to a chronic pericarditis. However the role of corticoids in chronic constrictive pericarditis is not known [7].

Many research are underway to identify means of preventing the progression of effusive tuberculous pericarditis to a constrictive phase. Ntsekhe et al have demonstrated a reduced level of N-acethyl-aspartyl-lysyl-proline (Ac. SDKP) an antifibrinotic protein in the pericardial fluids in patients with tuberculous pericarditis. On the other hand Mnguni et al found that angiotensin converting enzyme inhibitors (ACEI) were associated with an increased levels of Ac. SDKP in human plasma. These findings suggest a potential role of ACEI therapy in preventing tuberculous constrictive pericarditis [8].

Futhermore, there is some evidence that intra pericardial fibrinolytic agents in patients with purulent and tuberculous pericarditis can prevent progression to a constrictive phase [9].

Despite medical treatment, 50\% develop constriction and the patients do poorly without pericardiectomy. Early pericardiectomy is recommended in order to improve long term survival. It is usually reserved for effusive/constrictive disease or recurrent effusions [10].

\section{Conclusions}

Constrictive pericarditis is an uncommon disease. Tuberculous pericarditis is a rare cause in the united states, but remains the most common cause in some parts of the world. In third world countries this is the most common cause of right heart failure The diagnosis is challenging especially in PPD negative patients. The treatment is mainly curative and thus an aggressive approach in diagnosis and therapy is mandatory. New potential strategies for preventing tuberculous constrictive pericarditis have recently emerged but further investigations are necessary in order to confirm their efficacy. 


\section{References}

[1] Nishimura RA. Constrictive pericarditis in the modern era: a diagnostic dilemma - Heart. 2001 (6):619-623.

[2] Osterberg L, Vagelos R, Atwood JE. Case presentation and review: constrictive pericarditis. Osterberg, West J Med. (4):232-239.

[3] Ntsekhe M, Shey Wiysonge C, Commerford PJ, Mayosi BM. The prevalence and outcome of effusive constrictive pericarditis a systemic review of literature. Cardiovasc J Afr 2012, Jan 12;22:1-5. doi: 10.5830/CVJA-2011-072.

[4] Feigenbaum's- Echocardiography, seventh edition, pages 256-259, 556-558.

[5] Grossman's Cardiac Catheterization, Angiography, and Intervention, pages 731-736.

[6] CinarB, EncY, GokselO et al:Chronic constrictive tuberculous pericarditis:Risk factors and outcome of pericardiectomy. IntJ Tuberc lung Dis 2006; 10:701-706.

[7] Ntsekhe M, Wiysonge C, Volminky H, Commerford PY, Mayosi BM. Adjuvant corticosteroids for tuberculous pericarditis promising but not proven. QJM 2003;96:593-599.

[8] MutyabaA, MayosiBM. JACC; 2016. Emerging countries and tuberculosis constriction. JACC; 2016.

[9] Cui HB, Chen XY, Cui CC, Ochodo EA et al. The efficacy and safety of complete pericardial drainage by means of intrapericardial fibrinolysis for the prevention of complications of pericardial effusion:A systemic review protocol. BMJ open 2016;6:e07842.

[10] Bozbuga N, Erentug V, Eren E, Erdogan HB, Kirali K, Antal A, Akinci E, Yakut C. Pericardiectomy for chronic constrictive tuberculous pericarditis. Tex Heart Inst J. 2003;30 (3):180-185.

[11] Mnguni AT, Engel ME, Borkum MS, Mayosi BM. The Effects of Angiotensin Converting Enzyme Inhibitors (ACE-I) on Human N-Acetyl-Seryl-Aspartyl-Lysyl-Proline (Ac-SDKP) Levels: A Systematic Review and Meta-Analysis. PLoS One 2015;10:e0143338.

[12] Kakia A, Wiysonge CS, Ochodo EA, Awotedu AA, Ristic AD, Mayosi BM. The efficacy and safety of complete pericardial drainage by means of intrapericardial fibrinolysis for the prevention of complications of pericardial effusion: a systematic review protocol. BMJ open 2016;6:e007842. 